
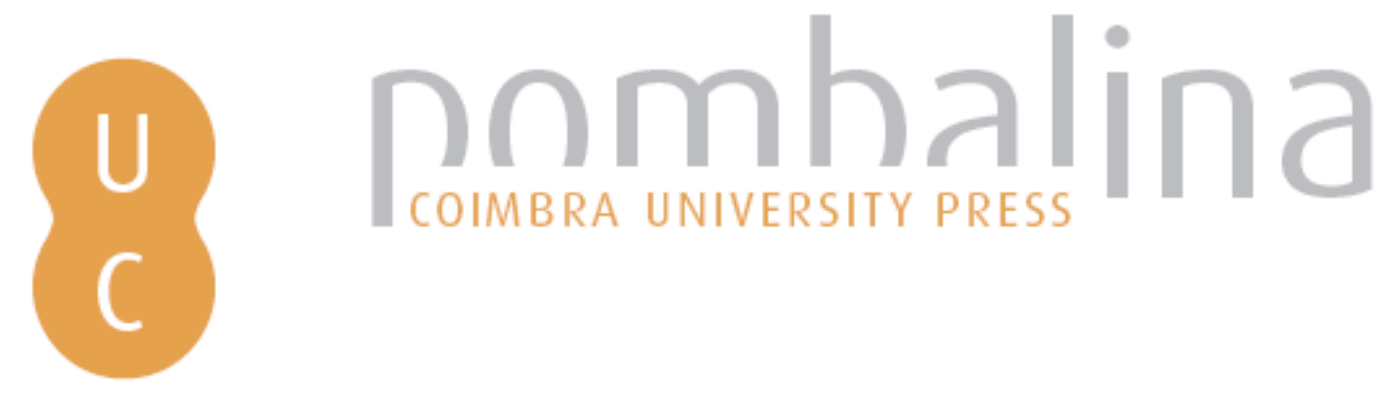

\title{
La enseñanza universitaria asociada con la extensión: el aprendizaje en servicio: una propuesta para la formación en ciencia de la información
}

\author{
Autor(es): $\quad$ Coringrato, Marcela; Carolina Rojas, Maria; Sleimen, Silvia \\ Publicado por: Imprensa da Universidade de Coimbra \\ URL \\ persistente: URI:http://hdl.handle.net/10316.2/31960 \\ DOI: DOI:http://dx.doi.org/10.14195/978-989-26-0319-3_36 \\ Accessed : $\quad$ 26-Apr-2023 13:43:02
}

A navegação consulta e descarregamento dos títulos inseridos nas Bibliotecas Digitais UC Digitalis, UC Pombalina e UC Impactum, pressupõem a aceitação plena e sem reservas dos Termos e Condições de Uso destas Bibliotecas Digitais, disponíveis em https://digitalis.uc.pt/pt-pt/termos.

Conforme exposto nos referidos Termos e Condições de Uso, o descarregamento de títulos de acesso restrito requer uma licença válida de autorização devendo o utilizador aceder ao(s) documento(s) a partir de um endereço de IP da instituição detentora da supramencionada licença.

Ao utilizador é apenas permitido o descarregamento para uso pessoal, pelo que o emprego do(s) título(s) descarregado(s) para outro fim, designadamente comercial, carece de autorização do respetivo autor ou editor da obra.

Na medida em que todas as obras da UC Digitalis se encontram protegidas pelo Código do Direito de Autor e Direitos Conexos e demais legislação aplicável, toda a cópia, parcial ou total, deste documento, nos casos em que é legalmente admitida, deverá conter ou fazer-se acompanhar por este aviso.

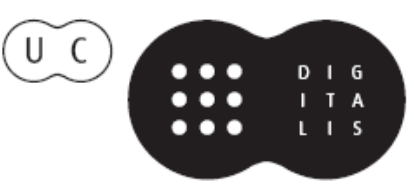


Maria Manuel Borges

Elias Sanz Casado

Coordenação

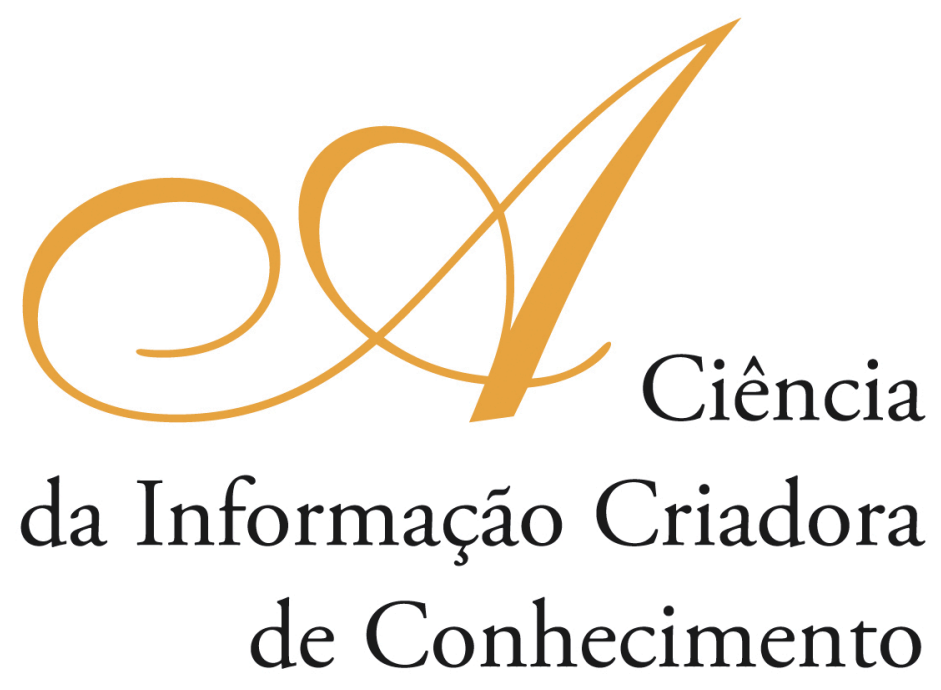

Vol. I

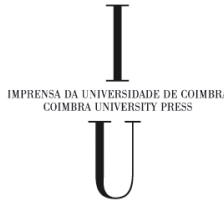

- COIMBRA 2009 


\title{
LA ENSEÑANZA UNIVERSITARIA ASOCIADA CON LA EXTENSIÓN: \\ EL APRENDIZAJe EN SERVICIO: UNA PROPUESTA PARA \\ LA FORMACIÓN EN CIENCIA DE LA INFORMACIÓN
}

\author{
Marcela Coringrato \\ Universidad Nacional de Mar del Plata (Argentina) \\ Maria Carolina Rojas \\ Universidad Nacional de Mar del Plata (Argentina) \\ Silvia Sleimen \\ Universidad Nacional de Mar del Plata (Argentina)
}

\section{Resumen}

Se destaca la importancia de la vinculación entre la universidad y la comunidad. Se aportan algunos conceptos centrales acerca de la misión social de la educación superior con especial énfasis en el informe a la Unesco sobre la Educación Superior del ańo 1991 y la Conferencia Regional de Educación Superior del año 2008. Se define la metodología del aprendizaje-servicio mediante la cual los jóvenes desarrollan sus conocimientos y competencias a través de una práctica de servicio a la comunidad. Se plantea el paradigma de la cognición situada como sustento a la metodología del aprendizaje-servicio. Se expone una propuesta para la práctica profesional de los estudiantes de ciencia de la información basada en los principios de la metodología descripta, cuya característica principal es su desarrollo a lo largo de toda la carrera. Se explican cada uno de los pasos del proyecto en base a la propuesta del Ministerio de Educación de la República Argentina para el desarrollo de proyectos de aprendizaje-servicio. Se plantean conclusiones.

\begin{abstract}
The relationship between the university and the comunity is of considerable importance in the service learning methodology. Key concepts about the social function of high education are provided, with special emphasis in the ALA Unesco report from 1991 and The Regional High Education Conference from 2008. Service learning methodology through which students develop their knowledge and competence is defined in terms of a service learning practice. A proposal aimed at information science students for their professional practice is formulated, based on the principles of the described methodology. This practice is developed throughout their career. Each step of the project is explained on agreement with the "Ministerio de Educación de la República Argentina" for the development of service learning project. The conclusions are presented.
\end{abstract}

\section{Introducción}

El glosario de términos en educación superior señala que la Extensión Universitaria es una de las funciones sustantivas de la universidad que tiene como finalidad propiciar 
y establecer procesos permanentes de interacción e integración con las comunidades nacionales e internacionales, en orden a asegurar su presencia en la vida social y cultural del país, a incidir en las políticas nacionales y a contribuir a la comprensión y solución de sus principales problemas. Comprende los programas de educación permanente, cursos, seminarios y demás programas destinados a la difusión de los conocimientos y al intercambio de experiencias. Comprende además, las actividades de servicio tendientes a procurar el bienestar general y la satisfacción de las necesidades de la sociedad. ${ }^{1}$

Esta característica fue instaurada en las universidades latinoamericanas con el establecimiento de la Reforma Universitaria, en 1918; en ese momento, sus inspiradores decidieron tomar para las casas de educación superior la misión de cobrar significado en su tiempo y en su lugar de existencia, intentando dar "respuestas adecuadas a las nuevas situaciones espirituales y sociales. Este requerimiento constituye el hecho más sorprendente y significativo en la historia de las universidades latinoamericanas". 23

A pesar de la equiparación institucional de esta actividad con la enseñanza y la investigación, distintas razones la mantienen relegada en la práctica cotidiana de sus actores. Como consecuencia del escaso vínculo entre la Universidad y su entorno sus prácticas son en muchos casos socialmente ineficientes.

Un desafío para la educación superior es buscar nuevas formas de práctica de integración social que permita generar un mayor impacto de sus actividades en la satisfacción de las necesidades comunitarias y en la inserción de sus egresados.

\section{La Misión Social de la Educación Superior}

Argentina, integrante de lo que se ha dado en llamar países emergentes, vive una realidad profundamente inequitativa. Las diferencias en las oportunidades que reciben sus habitantes son evidentes. Del mismo modo las desigualdades y contradicciones impiden el crecimiento y desarrollo para la mayoría de los países de América Latina. Hoy esta región es la más desigual del planeta.

Para superar este escenario es necesario que se materialicen una serie de transformaciones, entre las cuales, la educación tiene un espacio protagónico. Y, en lo que compete a la Educación Superior deberá asumir su responsabilidad en un mundo donde el conocimiento constituye un elemento insustituible para el avance social.

En América Latina "se necesita una educación que contribuya eficazmente a la convivencia democrática, a la tolerancia y a promover un espíritu de solidaridad y de cooperación; que construya la identidad continental; que genere oportunidades para

\footnotetext{
${ }^{1}$ Glosario de términos en educación superior. (n.d.). Consultado en: http://www.iesalc.unesco.org.ve/ programas/glosarios/Glosario\%20SUE-Colombia.pdf. Fuente: Ley 30 de 1992. Título Sexto 'Disposiciones Generales, Especiales y Transitorias'.Cap. I. 'Disp. Grales'

2 Romero, José Luis. (1959) Función social de la universidad latinoamericana. Consultado en: http:// www.iesalc.unesco.org.ve/pensamientouniv/argentina_drt/romero_jose-luis.pdf

3 Tünnermann, Carlos. (n.d.) El nuevo concepto de la extensión universitaria. Consultado en http://www. uc.edu.ve/descouc/cuaderno5.htm (Consulta: 13/06/06)
} 
quienes hoy no las tienen y que contribuya, con la creación del conocimiento, a la transformación social y productiva de nuestras sociedades"”.

La Educación Superior es un derecho humano y es responsabilidad de las comunidades académicas definir los principios básicos en los cuales se fundamenta la formación de los ciudadanos. Además debe garantizar el acceso y permanencia en condiciones equitativas y con calidad para todos y todas, integrando todos los sectores sociales e incorporar el diálogo de saberes y el reconocimiento de la diversidad de valores y modos de aprendizaje.

Asimismo, debe estar orientada a la educación integral de las personas, fijándose como prioridad la formación de un profesional-social, capaz de abordar con responsabilidad ética su inserción en el medio, participando activamente de la construcción de una sociedad más justa. En síntesis, tiene un compromiso vital, que incluye la promoción del respeto y la defensa de los derechos humanos; la lucha por la igualdad y la justicia social; el fomento de una cultura de paz.

Estos compromisos deben expresarse en todos los programas de formación, así como en las prioridades de investigación, extensión y gestión.

Para ello se debe avanzar en la creación de una relación más activa con sus contextos asumiendo la responsabilidad con el desarrollo sostenible de la sociedad. Ello exige "impulsar un modelo académico caracterizado por la indagación de los problemas en sus contextos; la producción y transferencia del valor social de los conocimientos; el trabajo conjunto con las comunidades; una investigación científica, tecnológica, humanística y artística fundada en la definición explícita de problemas a atender, de solución fundamental para el desarrollo del país o la región, y el bienestar de la población; una activa labor de divulgación, vinculada con la creación de conciencia ciudadana sustentada en el respeto a los derechos humanos y la diversidad cultural; un trabajo de extensión que enriquezca la formación, colabore en detectar problemas para la agenda de investigación y cree espacios de acción conjunta con distintos actores sociales, especialmente los más postergados"

Gran parte del cumplimiento de esta tarea corresponde a la asunción, por parte de las universidades, de su misión social. Y esto consiste, al decir de Risieri Frondizi, en ponerse al servicio del país. "Una universidad puede formar profesionales excelentes, aunque socialmente inútiles".

No es menos importante subrayar que la mayoría de la población carece de los servicios de aquellos profesionales que se formaron gracias al esfuerzo de toda la comunidad.

Es urgente promover una reforma que permita usar el conocimiento de manera útil, pertinente y equitativa. La Universidad debe pensar no sólo en la instrucción profesional sino también en la formación personal de sus estudiantes, como lo declaman sus estatutos.

En este contexto surge el concepto de Responsabilidad Social Universitaria como un desafío a formar personas sensibles a los problemas de los demás, comprometidas

\footnotetext{
${ }^{4}$ Conferencia Regional de Educación Superior (2008).

5 Conferencia Regional de Educación Superior (2008)

${ }^{6}$ Frondizi, Risieri (2005)
} 
con el desarrollo de su pueblo y con la inclusión social de los más vulnerables, capaces de integrar un proyecto personal con un proyecto de transformación y enriquecimiento comunitario.

\section{El aprendizaje-servicio}

"El aprendizaje-servicio podría definirse en primera instancia, como una metodología de enseñanza y aprendizaje mediante la cual los jóvenes desarrollan sus conocimientos y competencias a través de una práctica de servicio a la comunidad."

Algunas de las características fundamentales de esta metodología son:

- Sostiene simultáneamente una intensión pedagógica y una intensión solidaria. Los destinatarios del proyecto son la población atendida y los estudiantes, ya que ambos se benefician. El énfasis está puesto en la adquisición de aprendizajes y en el mejoramiento de las condiciones de vida de la comunidad concreta.

- Es vital el protagonismo de los estudiantes. Es una propuesta de aprendizaje activo $y$, por lo tanto, deben ser los estudiantes quienes deben protagonizar y hacer propias las actividades. Si los estudiantes no se involucran, el impacto en los aprendizajes no es el mismo.

- Las actividades se orientan a colaborar eficazmente con la solución de problemáticas comunitarias concretas. En este sentido, la acción transformadora ocurre en todos los actores involucrados. Los estudiantes resignifican sus aprendizajes, participan en la construcción del conocimiento, desarrollan habilidades críticas y reflexivas, revalorizan el conocimiento no disciplinar. Los integrantes de la comunidad encuentran un ámbito donde plantear sus demandas genuinas; son participantes activos de los procesos transformadores de su realidad; esta participación activa crea un espacio de encuentro, que elimina el riesgo de reforzar las inequidades que corren otras prácticas donde los destinatarios juegan un rol pasivo.

- Las prácticas solidarias están estrechamente vinculadas con los contenidos curriculares. La planificación pedagógica es precisamente lo que distingue al aprendizajeservicio de otras prácticas de extensión, voluntariado, iniciativas solidarias, etc.

El aprendizaje-servicio es una metodología que promueve el desarrollo de competencias a través de actividades de servicio a la comunidad, basándose en la convicción de que puede ser un factor de transformación de la realidad social. El aprendizaje-servicio es una metodología para una práctica, que subraya el valor de las actividades educativas solidarias al servicio de la comunidad y desarrolla su potencial formador conectándolas con el aprendizaje formal.

Por otra parte, permite a los estudiantes adquirir una mejor comprensión del contenido académico, aplicando conocimientos en beneficio de su comunidad. Los estudiantes, en definitiva, aprenden en el marco de experiencias de servicio a la comunidad, comprobándose que el aprendizaje-servicio contribuye tanto a la formación académica y ética de los estudiantes como a la resolución de problemas genuinos de una sociedad.

\footnotetext{
7 Tapia, María Nieves (2000)
} 
Es importante hacer aquí alguna referencia a los principios del paradigma de la cognición situada. Esta se funda en la premisa de que el conocimiento es situado, es parte y producto de la actividad, el contexto y la cultura en que se desarrolla y utiliza. Reconoce que el aprendizaje es, ante todo, un proceso de enculturación en el cual los estudiantes se integran gradualmente a una comunidad o cultura de prácticas sociales. Aprender y hacer son acciones inseparables, y, además, se debe aprender en el contexto pertinente.

La forma tradicional de enseñanza se traduce en aprendizajes poco significativos, es decir, carentes de sentido y aplicabilidad, y en la incapacidad de los alumnos por transferir y generalizar lo que aprenden. En la cognición situada la enseńanza se centra en prácticas educativas coherentes, significativas y propositivas. Además su autenticidad puede determinarse por el grado de relevancia cultural de las actividades en que participa el estudiante, así como por el tipo y nivel de actividad social que éstas promueven. En este paradigma la unidad básica de análisis no es el individuo en singular ni los procesos cognitivos, sino la acción recíproca, es decir, la actividad de las personas que actúan en contextos determinados. En definitiva, el aprendizaje se entiende como los cambios en las formas de comprensión y participación de los sujetos en una actividad conjunta.

Una de las estrategias de la enseñanza situada es, precisamente, el aprendizaje basado en el servicio a la comunidad (service learning), cuyas principales características son:

- Los estudiantes aprenden y se desarrollan mediante la participación activa en experiencias de servicio cuidadosamente organizadas que responden a las necesidades de la comunidad.

- Integrado dentro del currículo académico, proporciona al alumno un tiempo para pensar, hablar o escribir acerca de lo que hace y observa durante la actividad.

- Proporciona la oportunidad de aplicar los conocimientos en situaciones de vida real.

- Fortalece las enseñanzas del sistema educativo extendiendo el aprendizaje del alumno hacia la comunidad, y ayuda a fomentar un sentido de responsabilidad y cuidado hacia los demás. ${ }^{8}$

Una de sus importantes consecuencias es que puede influir en el sentido de identidad y justicia social de los adolescentes y jóvenes. Representa una oportunidad de reorientar la cultura contemporánea hacia el valor de ayudar a los demás y de asumir nuestra responsabilidad social. Enfatiza la ayuda a los otros, no como caridad o lástima, sino como responsabilidad social relacionada con una toma de conciencia moral, social y cívica.

Como puede verse, el paradigma de la cognición situada enmarca y da sustento a la metodología de aprendizaje-servicio.

Desarrollo del proyecto para la Práctica Profesional

Cada año se comunican con el Departamento de Documentación varias entidades (sociedades de fomento, centros barriales, etc.) solicitando asesoramiento y asistencia

\footnotetext{
${ }^{8}$ Díaz Barriga Arceo, Frida (2003)
} 
para el desarrollo de una biblioteca. En la mayoría de los casos no es posible atender estos pedidos. Si bien no es viable la implementación completa de una biblioteca y su gestión realizando sólo algunas prácticas por asignatura, sí es factible la realización de acuerdos donde el trabajo de los estudiantes se vería complementado con pasantías (también de estudiantes, en este caso voluntarias) que, además de su reconocimiento para la práctica profesional, supondrían una certificación de experiencia profesional ${ }^{9}$.

La práctica profesional que proponemos consiste en que las asignaturas, a lo largo de toda la carrera, efectúen, cada una en la medida de sus posibilidades y de acuerdo con una evaluación previa, por lo menos una de las prácticas habituales de aula en una unidad de información real. Para esto el Departamento, junto con los docentes de la Práctica Profesional, elegirá antes del inicio de la actividad académica dicha unidad de información, a fin de que se ajuste al espíritu del proyecto. La organización seleccionada podría rotarse cada 3 años con el fin de que todos los alumnos, en cada materia, tengan un contacto efectivo con ella.

De esta forma, la práctica profesional sería paralela al desarrollo de toda la carrera, y se intensificaría en la medida en que el alumno avanza hacia su graduación. La evaluación de estas prácticas por asignaturas estaría a cargo tanto de los docentes de cada materia, como de los docentes de la Práctica Profesional. En lo concerniente a la asignatura, cada profesor estaría en libertad de calificarla de acuerdo con su criterio en cuanto al cumplimiento de los objetivos académicos. Respecto a la práctica profesional, se evaluarían integralmente el cumplimiento de los objetivos que exceden lo académico y se registraría en un historial individual de cada alumno, que se construiría a lo largo de toda la carrera. Al finalizar los tres ańos de práctica, el alumno cumpliría con las condiciones que establece la práctica actual, pero ya con un profundo conocimiento de la institución, de sus usuarios, de su entorno. No es necesario subrayar que la experiencia que de esta manera adquirirían los alumnos supera enormemente a la que se cumple actualmente. Sí es importante destacar que el éxito de esta práctica depende en gran medida del compromiso que cada docente adquiera con el proyecto.

La implementación de un proyecto de estas características supone recorrer una serie de pasos. Además de estas instancias que se desarrollan en un orden secuencial, se recomienda un conjunto de acciones que atraviesan todo el proceso y que tienen por finalidad evaluar periódicamente lo actuado, internalizar los conocimientos adquiridos, medir el grado de ajuste a los objetivos planteados, realizar cambios sobre la marcha, etc.

Estas acciones son:

1. Reflexión: si bien la reflexión se da naturalmente en todo el proceso a través del acompañamiento del docente, conviene plantear espacios concretos para trabajarla en forma sistemática. Algunos de los aspectos para reflexionar pueden ser el reconocimiento de la vinculación de la actividad con los aprendizajes; el impacto en las vivencias personales; autoevaluación del desempaño personal; registro de opiniones de los destinatarios del proyecto.

\footnotetext{
9 Creemos, asimismo, que estableceríamos de esta manera la necesidad de, al finalizar el período estipulado en el acuerdo, contratar ahora si un pasante rentado, o un graduado, para el funcionamiento pleno de la unidad de información. Con lo cual otra de las consecuencias de esta práctica sería la creación de fuentes de trabajo.
} 
2. Sistematización y comunicación: es conveniente recolectar datos, anécdotas, experiencias, a fin de construir el registro de lo actuado. Permite detectar aciertos y debilidades de la experiencia y evitar que muchos aportes se pierdan. Por otra parte, debe mantenerse una comunicación fluida entre los diversos participantes, haciendo circular la información, y definiendo canales para difundir los logros, ajustes, modificaciones, errores. La transformación de conocimiento tácito en explícito posibilita, además, que futuros interesados -estudiantes, docentes- accedan a esas prácticas como antecedentes empíricos que pueden capitalizarse.

3. Evaluación: este aspecto es central dentro del proyecto y consiste en un proceso de reflexión permanente. Es uno de los desafíos más importantes, pues hay una seria tendencia a convertirla en una formalidad. Hay que tener en cuenta que esta experiencia no consiste sólo en la adquisición de conocimientos "duros", sino que apunta a la transformación interior de todos los actores y a la adquisición de habilidades para la resolución de problemas reales. Por lo tanto hay que evaluar tanto el servicio solidario desarrollado como el impacto del proyecto en la dimensión personal de cada estudiante y los aprendizajes involucrados. Algunas recomendaciones para una evaluación efectiva son: considerar aspectos cualitativos (competencias desarrolladas, concientización adquirida por el grupo sobre los problemas sociales, impacto del proyecto en cada estudiante en cuanto a la elevación de autoestima, seguridad y confianza en sus propias capacidades); efectuarla en forma participativa y democrática; atender al proceso y no sólo a los resultados; precisar el impacto en los destinatarios; medir la flexibilidad con la que se cumplió la planificación; etc.

Ahora si, las etapas a desarrollar son:

A. Diagnóstico y planificación.

a. Paso 1. Conocimiento y comprensión del concepto de aprendizaje-servicio y motivación.

La motivación es el secreto del éxito de todo cuanto se emprende. También lo es en el caso de un programa de aprendizaje-servicio. Cuando éste surge de una iniciativa de los alumnos, o a pedido de la comunidad, pero involucrando fuertemente en el desarrollo a los estudiantes, la motivación existe previamente. En nuestro caso, al tratarse de un requisito de la Carrera, se corre el serio riesgo de que los alumnos no se sientan protagonistas de la tarea solidaria y de que no se involucren a fin de lograr los objetivos que se persiguen en relación con la formación ética. Por esta razón será doblemente importante en esta instancia prever encuentros iniciales para concientización, comprensión cabal de la metodología, capacitación, participación de lecturas apropiadas, relatos de experiencias similares, etc.

b. Paso 2. Diagnóstico participativo.

En el caso de los proyectos que responden a una demanda concreta de la comunidad, o los que se gestionan alrededor de una inquietud de los estudiantes, esta etapa supone un fuerte trabajo de relevamiento de la realidad que consiste en recabar información y consultar con los grupos e instituciones participantes. El diagnóstico pretende identificar las necesidades reales de la comunidad que puedan ser atendidas desde la institución educativa, los recursos humanos y materiales que permitan desarrollar un proyecto y la oportunidad para el aprendizaje significativo de los estudiantes. En nuestro caso, se trata específicamente de colaborar con la implementación y/o gestión de una unidad de información. Por esta razón, el diagnóstico se circunscribirá a 
relevar toda la información necesaria para evaluar la factibilidad de la UI, es decir, las posibilidades edilicias, técnicas, materiales, de personal, etc. o la situación existente en un centro en funcionamiento. Esta tarea será previa al trabajo concreto de los alumnos, es decir, corresponde al Departamento y a la cátedra Práctica Profesional y constituye la instancia en que se elige concretamente el destinatario de la acción. Una vez que se ha determinado dónde se realizarán las prácticas y se han formalizado los acuerdos correspondientes con la institución, el trabajo conjunto a realizar con los estudiantes es:

i. de identificación y análisis crítico de la necesidad/problema/desafío barrial o comunitario.

Aquí será necesario determinar los recursos disponibles (humanos y materiales), si ya se ha iniciado un trabajo previo, si hay más de una institución involucrada, si el pedido de colaboración responde a una necesidad sentida por la comunidad o hay que trabajar para que lo considere un logro a conseguir, una meta a alcanzar, un compromiso que requiere del acuerdo de todos. Se podría aplicar un análisis FODA para detectar cuál es el punto de partida, con qué contamos a favor de nosotros y qué acciones específicas deben implementarse.

ii. Análisis de la posibilidad de respuesta desde la institución educativa.

En función de nuestra identidad, la solución que estamos en condiciones de brindar es específica y concreta. Aún así, la experiencia indica que la intervención en una realidad careciente saca a la luz toda una serie de problemas sociales múltiples y complejos a los que no podremos dar respuesta. Eventualmente, el Departamento puede establecer vínculos con otras facultades, que permitan abordar la problemática desde distintos lugares.

Asimismo, es determinante para el éxito del proyecto, que el plan esté de acuerdo con los recursos, prioridades y tiempos disponibles. Es conveniente plantearse qué personal y materiales requerirá (por ejemplo, traslado), cómo se administrarán y qué obstáculos puede tener su implementación. De ellos deriva la viabilidad del proyecto. Sería importante considerar la posibilidad de establecer alianzas con otros organismos comunitarios o instituciones que puedan involucrarse estableciendo acuerdos de colaboración y participación mutua.

c. Paso 3. Diseño y planificación del proyecto.

El diseńo de un proyecto de aprendizaje-servicio es el proceso de elaboración de la propuesta de trabajo que articula una intencionalidad pedagógica y una intencionalidad solidaria. Por eso incorpora las herramientas básicas de la planificación pedagógica y algunas cuestiones referidas a la ejecución de proyectos sociales.

\section{Fundamentación}

Como ya se ha expuesto, la Práctica Profesional actualmente vigente, no responde a las necesidades que presentan los graduados al momento de entrar en el mercado laboral. Esta inquietud encuentra en el marco de una Reforma de Planes de Estudio el momento adecuado para su redefinición.

Por otra parte, nuestra profesión se ha caracterizado tradicionalmente por contar con un alto componente ético, pero las nuevas circunstancias otorgan a este asunto 
una consideración particular sobre todo en los últimos años, como lo demuestra la adopción de un código de ética en muchos países, el aumento de publicaciones sobre el tema y la incorporación de esta materia en las currículas de las carreras universitarias vinculadas con gestión de información.

Sólo con mencionar algunos de los ámbitos donde han surgido profundas transformaciones como resultado de la explosión documental, la revolución de las TICs, la aparición de nuevos medios y canales de comunicación, se verá claramente que es indispensable su agregación: medios electrónicos; propiedad intelectual; privacidad y confidencialidad de la información; nuevas competencias profesionales; innovación tecnológica; uso, difusión y/o comercialización de la información; discriminación; etc.

En el contexto de estas dos necesidades, creemos que la metodología del aprendizajeservicio es la herramienta ideal para crear en nuestros estudiantes la conciencia del compromiso que nos compete como profesionales de la información, a la vez de garantizar un ámbito de formación estrechamente vinculado con la realidad social de nuestros países.

\section{Objetivos del servicio solidario y del aprendizaje.}

La Educación Superior debe procurar desarrollar e integrar el dominio de un campo del saber o de competencias profesionales, el sentido crítico, la capacidad de reflexión y una actitud de servicio a la colectividad.

Objetivos Generales:

- Posibilitar al estudiante de Bibliotecología y Documentación familiarizarse con las funciones y las responsabilidades de un profesional de la información para el desempeño de su rol como un profesional social comprometido con su entorno.

- Comprender cada asignatura como una unidad de formación concreta para el desarrollo efectivo de las prácticas, mediante un proceso de enseńanza y de aprendizaje vinculado con el contexto de aplicación.

Objetivos específicos: los objetivos aquí descritos no detallarán conocimientos determinados a adquirir por los alumnos, pues éstos dependerán de la asignatura que estén cursando y del objetivo en particular de ese práctico.

Objetivos de aprendizaje:

- Que el alumno realice al menos un trabajo práctico por asignatura, por cuatrimestre, en la unidad de información propuesta, adquiriendo una competencia concreta vinculada con los contenidos mínimos de cada asignatura, evaluable por el docente.

- Que el alumno sea capaz de identificar qué competencia profesional se requiere para la resolución de cada problemática que se presente.

- Que el alumno adquiera la capacidad de integrar los conocimientos recibidos en cada asignatura en la resolución de las contingencias propias de una unidad de información.

Objetivos de servicio:

- Que la institución elegida, al finalizar la práctica profesional, cuente con una biblioteca organizada y en condiciones de responder las demandas propias de la comunidad. 
- Que los alumnos desarrollen un sentido de responsabilidad frente a las necesidades de comunidades carenciadas fundamentado en la noción de Compromiso Social Universitario.

- Que los alumnos tomen conciencia de su capacidad para transformar el entorno como futuros profesionales sociales.

- Que los alumnos reconozcan la necesidad y las ventajas del trabajo conjunto de la universidad con la comunidad.

- Que los alumnos valoren la adquisición de conocimientos no formales a través de la interacción con miembros de la comunidad a la que se dirige el servicio.

- Que los alumnos tomen conciencia de que se están formando en esta Universidad Pública gracias al esfuerzo de toda la comunidad, a la cual deben devolverle en servicios prestados.

\section{Destinatarios del servicio solidario.}

Los destinatarios de este proyecto de aprendizaje-servicio claramente son dos: nuestros alumnos, que se verán favorecidos en la adquisición de sus nuevos conocimientos, pero en un entorno real, es decir, aprendizajes significativos; y la comunidad usuaria de la unidad de información seleccionada, que tendrá acceso a los servicios de la biblioteca, y a los que pudieren surgir del contacto con la comunidad y de las alianzas que se gestionen con otras facultades.

Es importante aquí destacar que, indudablemente, también el cuerpo docente se verá ampliamente beneficiado por la experiencia, toda vez que creemos imposible compartir una práctica solidaria y emerger sin una visión diferente del mundo.

\section{Actividades del servicio solidario.}

La acción concreta que se llevará a cabo es la implementación y puesta en marcha de una biblioteca. En tanto la elección de la futura unidad de información es posterior a la descripción de este proyecto, y rotativa, no es posible determinar en esta instancia en qué estado se hallará, ni otras particularidades que definirán los cursos de acción a seguir. Pero sí es importante dejar sentado que esta etapa (la selección de las actividades con vistas a lograr aprendizajes significativos y un servicio eficaz) debe desarrollarse antes de la puesta en marcha y una vez seleccionado el lugar objeto del servicio.

\section{Contenidos y actividades del aprendizaje.}

$\mathrm{Al}$ igual que en el punto anterior, aquí no es posible aún explicitar la vinculación curricular del proyecto. Al momento de realizarse la práctica, cada asignatura deberá enumerar con claridad los contenidos que se aprenderán y se pondrán en juego con su ejecución. Hay que destacar que el desarrollo de cada proyecto, que corresponderá a cada unidad de información nueva, deberá incluir la identificación de las asignaturas 
y áreas del conocimiento participantes y también indicar de la forma más concreta posible cada contenido de aprendizaje en sí. También se añadirá el informe final de las materias, con resultados de cada práctica, alumnos participantes, evaluaciones realizadas, y toda otra información de interés, para construir, no sólo el informe de la práctica, sino el historial personal de cada alumno a lo largo de su carrera.

\section{Tiempos-Cronograma tentativo.}

La distribución temporal se puede exponer en un cronograma compatible con el Plan de Estudios. Idealmente, se construirán dos tipos de cronogramas: uno general, correspondiente a todo el proyecto, y que inicialmente se desarrollará en tres años; y otros parciales, que corresponderán a cada asignatura en cada cuatrimestre. Los docentes de la Práctica tendrán la responsabilidad de que todos sean coherentes entre sí y que se estructuren en una línea de acción coordinada.

VII. Responsables y protagonistas.

Se pueden distinguir varios responsables y protagonistas en este proyecto:

- los estudiantes que deberán participar de cada paso del proyecto, desde el diagnóstico a la evaluación. Quedará registrado cuántos participan y desde qué asignatura.

- los docentes, tanto quienes son responsables del proyecto (Dirección del Departamento y Cátedra Práctica Profesional) como quienes apoyan desde el desarrollo de contenidos curriculares o acompañan las acciones.

- los miembros de las organizaciones de la sociedad civil, barriales, etc. donde estén insertas las bibliotecas, especialmente si han de tener alguna responsabilidad específica y si se han firmado acuerdos de trabajo conjunto.

- la comunidad en donde la unidad de información está inserta.

\section{Evaluación del diseño y coherencia interna del proyecto.}

Una vez elegida la unidad de información, y redactado el proyecto particular para ese destinatario, se debe realizar un análisis final que permitirá, a quienes están involucrados en su diseńo, evaluar si se han cumplido los pasos considerados como necesarios y si se ha tenido en cuenta el espíritu del aprendizaje-servicio: la participación de todos los sectores, el protagonismo estudiantil, el reconocimiento de las necesidades reales y sentidas de la comunidad, el planteo de un servicio solidario efectivo, la articulación plena con los aprendizajes curriculares.

Como ya se ha mencionado en varias oportunidades, el presente diseño no contempla la explicación de todas las instancias, atento ser marco para el desarrollo de los proyectos puntuales. Por esta razón no es posible aquí cumplir con las indicaciones planteadas en este apartado. Sí queremos precisar que es conveniente dedicar un espacio para asegurar la relación entre los distintos objetivos, las actividades concretas (que no están enumeradas en esta instancia) y los resultados esperados.

Además, se recomienda analizar la coherencia interna del proyecto. Esto significa considerar si hay adecuación y pertinencia en conceptos y actividades, en función de la fundamentación, los objetivos, las actividades, la evaluación y los resultados. 


\section{B. Ejecución del proyecto.}

La ejecución constituye la puesta en práctica de lo considerado en el proyecto y depende del diseńo del mismo. Se hace efectiva mediante la aplicación de diversas herramientas que facilitan, y a la vez permiten monitorear la marcha de las actividades y desarrollar el proceso de reflexión permanente y específica.

a. Paso 4. Establecimiento de alianzas barriales/institucionales y obtención de recursos.

Con respecto a los recursos, la herramienta para el monitoreo de su utilización será una planilla de rendición de cuentas.

En cuanto a las alianzas, se establecerán acuerdos, o se firmarán convenios con la institución objeto de la práctica. Pero, por otra parte, y como ya se mencionó anteriormente, es deseable formalizar vínculos con otras instituciones en función de sumar esfuerzos y crear sinergia. En todos los casos, los acuerdos decididos aportarán mayor solidez al proyecto y generarán articulaciones en "red" las que constituyen, en sí mismas, un aporte a la construcción comunitaria.

b. Paso 5. Implementación y gestión del proyecto de servicio solidario y desarrollo de los contenidos de aprendizajes asociados.

La puesta en marcha del proyecto supone que todos los actores involucrados participen de una red de trabajo que hace efectivas las actividades previstas. Es importante subrayar aquí que el éxito depende de el establecimiento de estrategias que apunten al aprendizaje, la reflexión que haga consciente los aprendizajes y garantice la retroalimentación, la sistematización y documentación de la experiencia, que permitirá las correcciones futuras, el seguimiento de los aprendizajes y del servicio, la contabilidad transparente y la buena planificación.

C. Evaluación y sistematización final.

a. Paso 6. Evaluación de la factibilidad de continuidad y multiplicación de los proyectos de aprendizaje-servicio.

La evaluación es central dentro de todo proyecto. Esta evaluación final debe realizarse aunque se hayan hecho otras durante el proceso. En esta instancia deberán registrarse todos los datos recabados durante la realización (número de participantes, registros administrativos, modalidades de participación de cada uno de los actores involucrados, etc.), como así también la evaluación específica de los aprendizajes curriculares, del servicio realizado, del nivel de logro de los objetivos, de los obstáculos encontrados. Debe también registrarse la percepción y las opiniones de los destinatarios.

En la sistematización final se procura darle un cierre reflexionando sobre el aprendizaje-servicio, comprendiendo y analizando lo realizado, para aprender de la práctica. Es indispensable para los participantes porque dimensionan realmente su tarea, les permite registrar su compromiso y las acciones desarrolladas, y funciona como un "escalón" para pensar nuevos proyectos. Es conveniente que esta instancia termine con el desarrollo de un producto que registre la experiencia (video, libro, etc.).

Es vital compartir la experiencia realizada. Para la institución es una forma de dar visibilidad a su compromiso y el de sus estudiantes, favoreciendo el reconocimiento externo y la autoestima de los participantes. La multiplicación es una forma de 
compartir el conocimiento, de brindar ideas y ejemplos a otras instituciones y de socializar las prácticas.

b. Paso 7. Celebración y reconocimiento a los protagonistas.

El reconocimiento es fundamental para fortalecer la autoestima de los participantes y para alentar la continuidad de estas prácticas. Es importante culminar la tarea y desarrollar un ritual de alegría que brote de las experiencias y vivencias de los jóvenes. La fiesta será así la consecuencia de algo importante que ha sucedido y es un momento de compartir y disfrutar más allá de la teoría, la reflexión y la evaluación.

\section{Conclusiones}

Dice el Informe a la UNESCO ${ }^{10}$ que la violencia contradice la esperanza que tenemos algunos en el progreso de la humanidad. Los enfrentamientos que surgen a diario, basados en prejuicios desfavorables hacia los demás, se ven multiplicados a través de los medios de comunicación. Es menester esforzarse en cambiar esta realidad. ¿Puede la educación colaborar en evitar estos conflictos o solucionarlos de manera pacífica? En dicho informe proponen dos mecanismos: el descubrimiento gradual del otro y la participación en proyectos comunes.

El descubrimiento del otro sólo es posible con el descubrimiento previo de uno mismo. Recién ahí estaremos en condiciones de ponernos en lugar del otro, de desarrollar la empatía.

El trabajo mancomunado detrás de un proyecto permite superar los hábitos individuales y valorizar los puntos de convergencia, por encima de los aspectos que nos separan.

Creemos que a estas premisas aplicables a toda la humanidad hay que sumarle lo propio de nuestra disciplina. El Manifiesto de la Unesco sobre la Biblioteca Pública proclama su fe en la biblioteca como fuerza viva para la educación, la cultura y la información, y como agente esencial para el fomento de la paz y del bienestar espiritual a través del pensamiento de hombres y mujeres, dado que "la participación constructiva y la consolidación de la democracia dependen tanto de una educación satisfactoria como de un acceso libre y sin limites al conocimiento, el pensamiento , la cultura y la información ${ }^{11}$ ".

Considera que "La libertad, la prosperidad y el desarrollo de la sociedad y de los individuos son valores humanos fundamentales. Estos sólo podrán alcanzarse mediante la capacidad de ciudadanos bien informados para ejercer sus derechos democráticos y desempeñar un papel activo en la sociedad ${ }^{12}$ ".

No hay dudas de que jugamos un rol fundamental en el desarrollo de sociedades más justas. Y de que estamos en condiciones, desde el lugar que ocupamos, de crear las circunstancias para que esta convicción se multiplique y se haga efectiva en acciones

\footnotetext{
${ }^{10}$ Delors, Jacques y otros. (1996)

${ }^{11}$ Manifiesto de la Unesco sobre la Biblioteca Pública (1994)

12 Manifiesto. - op. cit.
} 
concretas. Sentir empatía no significa unirse al sufrimiento, sino crear las condiciones para que la desigualdad que lo provoca no se perpetúe. A esa tarea nos abocamos.

\section{Referências bibliográficas}

Caraggio, José Luis (coord.) y otros. (2001) Contribución al estudio del sistema universitario argentino. Buenos Aires: Miño y Dávila.

Centro de Desarrollo Docente. Pontificia Universidad Católica de Chile. (n.d.). Consultado en http://www.puc.cl/cddoc/index.php [Consulta 18/11/08]

Centro de Voluntariado del Uruguay (CVU). (n.d.). Aprendizaje-servicio: el diseño de proyectos. Consultado en www.lasociedadcivil.org/uploads/ciberteca/libro_3_final_editado.pdf [Consulta 12/11/08]

CLAYSS. Centro latinoamericano de aprendizaje y servicio solidario. (n.d.). Consultado en http://www.clayss.org.ar/index_nuevo.php [Consulta 12/11/08]

Conferencia Regional de Educación Superior (2008). Declaración Final de la conferencia Regional de Educación Superior en América Latina y el Caribe. Cartagena de Indias, Colombia. 4 al 6 de junio.

Delors, Jacques y otros. (1996) La educación encierra un tesoro : informe a la Unesco de la Comisión Internacional sobre la educación para el siglo XXI, presidida por Jacques Delors. - Madrid : Santillana-Unesco.

Díaz Barriga, Frida. (2003). Cognición situada y estrategias para el aprendizaje significativo. Revista Electrónica de Investigación Educativa, 5 (2). Consultado en http://redie.ens.uabc. $\mathrm{mx} / \mathrm{vol} 5 \mathrm{no} 2 /$ contenido-arceo.html [Consulta 12/11/08]

Fontaine, France y Bernhard, Paulette [para el] Programa General de Información y UNISIST. (1988) Directrices para la redacción de objetivos de aprendizaje en bibliotecología, ciencias de la información y archivistica. Paris: Unesco. (PGI-88/WS/10)

Frondizi, Risieri. (2005) Misión Social. La universidad en un mundo de tensiones: misión de las universidades en América Latina. Buenos Aires: Eudeba.

González, Alba; Giorgetti, Daniel. (n.d.). Educación Solidaria: Itinerario y herramientas para desarrollar un proyecto de aprendizaje-servicio. Ministerio de Educación. Programa Nacional Educación Solidaria. Consultado en http://www.me.gov.ar/edusol/catalogopublicaciones. html\#modcap

Manifiesto de la UNESCO sobre la Biblioteca Pública. (1994). Consultado en http://www. fundaciongsr.es/documentos/manifiestos/mani94es.htm [Consulta 12/11/08]

Tapia, María Nieves. (2000) La Solidaridad como Pedagogía. Buenos Aires: Ciudad Nueva. 